\title{
Human Factors and Errors in Security Aviation: An Ergonomic Perspective
}

\author{
Michelle S. F. Arcúrio ${ }^{\mathbb{D}},{ }^{1}$ Eliane S. Nakamura, ${ }^{2}$ and Talita Armborst ${ }^{1}$ \\ ${ }^{1}$ National Civil Aviation Agency, AVSEC Certification Technical Branch, Brasília, Brazil \\ ${ }^{2}$ National Civil Aviation Agency, AVSEC Quality Control Technical Branch, Brasília, Brazil \\ Correspondence should be addressed to Michelle S. F. Arcúrio; michelle.arcurio@gmail.com
}

Received 28 August 2018; Revised 17 November 2018; Accepted 29 November 2018; Published 13 December 2018

Academic Editor: Yair Wiseman

Copyright (c) 2018 Michelle S. F. Arcúrio et al. This is an open access article distributed under the Creative Commons Attribution License, which permits unrestricted use, distribution, and reproduction in any medium, provided the original work is properly cited.

In a previous study we have identified the incidence of human factors and errors in the security screening process, concerning the Brazilian civil aviation. This finding led to the analysis of labor activity in the security checkpoint from an ergonomic perspective. The objective of this study was to evaluate the various labor conditions in the security checkpoints of Brazilian airports, in order to promote safer and higher performance of the AVSEC professionals, as well as the security equipment. For this purpose, we analyzed 14 of 60 questions of a self-observation questionnaire based on the theory of Generic Error-Modelling System (GEMS) and the four themes about human factors recommended by the International Civil Aviation Organization (ICAO). The questionnaire was responded to by 602 AVSEC professionals who worked in the security checkpoints of 18 Brazilian airports. Our analysis focused on the preponderant indexes of each question, taking into account the sequential arrangement in which the questions were displaced in the questionnaire and the classification of human factors and errors.

\section{Introduction}

The importance of aviation security has changed dramatically in the last years. The attacks of September 11th affected the way that government, corporations, and the general public perceive security in civil aviation. Thus, all of them are faced with the challenge of maintaining standards for security while facilitating smooth and efficient travels. As a consequence of the new threat situation, large investments into technology have been made, such as the X-ray machines placed in the security checkpoints. The state-of-the-art Xray machines provide high resolution images, several image enhancement features, and automatic detection of explosive material. However, it is clear that the best technology is only as valuable as the humans that operate it [1].

The objectives of ergonomics refer to the transformation of work to adapt to the characteristics and variability of the individual and the production process, aiming to acquire well-being, safety, quality, and productivity [2]. Ergonomics deals with environmental conditions (lightening, temperature, and others), measurement of time to perform a task, and the resulting errors and also identifies whether the labor organization is compatible with the activities carried out in a certain labor context. The ergonomic action aims to improve the human activity in the productive process of a system, associating criteria of health, welfare, and productivity.

In the study described by [3], for the safe operation in a complex system, such as a nuclear power plant, the temperature, light, and interface are important subfactors of environment in ergonomics.

Moreover, [2] described the different forms of ergonomic interventions: physical ergonomics, which concerns the characteristics of human anatomy, anthropometry, physiology, and their relationship with the physical activity; cognitive ergonomics, which studies the mental processes such as perception, memory, reasoning and motor response, and their effects on human interactions. It also investigates mental workload, decision-making, specialized performance, interaction between man and computer, human reliability, stress, professional education, among others.

The conception of work presents a dialectical framework between the prescribed work and the effective action, 
the real work, of the professionals. Many actions executed by the professionals may complete the gaps of prescribed work that come out in the routine [2]. In its very essence, these gaps can reflect the incidence of human factors and errors.

Several human factors and ergonomics projects have been directed at responding to accidents (train wrecks, airplane crashes, nuclear plant mishaps) and natural disasters (hurricanes, earthquakes, floods). Although most of this work has not been motivated by the reality of terrorism or, in particular, by the attacks of $9 / 11$, the research of human factors and ergonomics is increasingly making the connection with some aspects related to terrorism. For instance, it was recently reported that, in Israel, the center of attention for security regulation is the human factor. While the aviation security has drawn most of the international attention, Israel has to deal with the public and private road traffic. Thus, the solutions for security issues should consider the interface between the multimodal transportations [4].

In a previous study, [5] revealed the incidence of human factors and errors in the security screening process performed in Brazilian airports. In this context, the analysis of labor activity in the security checkpoint of passengers, under an ergonomic perspective, allows the evaluation and rearrangement of various labor conditions, in order to promote safer and higher performance of the aviation security-AVSEC (safeguarding civil aviation against acts of unlawful interference)-professionals, as well as the security equipment.

Furthermore, the level of association between human errors and factors involved in security screening process of passengers in Brazilian airports has been identified [6]. Using the bivariate analysis reported by [5], the authors suggested that cognitive abilities, understood as the way the human brain processes information available in its surroundings, include vigilance, attention, interpretation of X-ray images and object recognition, classification, and decision-making.

A number of subjects are covered by ergonomics, based on its fundamental premise of transforming the labor context. One theme is the variability of real work situation, either related to the humans or the production process. For instance, variations in the labor activity performance may occur in the short term, after stressful situations, or in the end of a working day. As for the AVSEC, it is important to investigate how the variability related to the professionals (fatigue, personal background, age, reasoning, etc.) and the unpredictability of real situations impact on AVSEC professionals, since they may result in human factors and errors.

Anther theme covered by ergonomics is the human reliability, which "results from the different cognitive processes that are mediated by the competence of a group of workers, to act in a certain context and supported by a system of production and task" [2]. It is related to the premise of transforming the labor context as well.

\section{Methods}

Arcúrio et al. [5] have previously employed the theory of Generic Error-Modelling System (GEMS) and the four themes about human factors recommended by the International Civil Aviation Organization (ICAO) to elaborate a self-observation questionnaire, consisting of 60 questions. A total of 602 AVSEC professionals who worked in security checkpoints, in eighteen Brazilian airports, in the years of 2013 and 2014 responded to such questionnaire (Figure 1). The professionals were asked to qualify, by using a Likert scale, the frequency and the intensity level or their agreement with the assertions presented in the questionnaire. 46 out of 60 questions in the questionnaire were categorized into seven principal components (PC) by Principal Component Analysis (PCA). The data variance was able to explain $42.04 \%$ of these PC, that is, the human factors and errors that were searched.

For its turn, the current study analyzed the other 14 questions (Table 1) of the total of 60, which have not yet been covered by [5]. This analysis focused on the preponderant indexes of each question, taking into account the sequential arrangement in which the questions were displaced in the questionnaire, and the classification of human errors developed by [7] or human factors presented in DOC 9808 [8].

\section{Results}

This section presents the questionnaire results in terms of the relative frequency for each group of questions, followed by a short description of the findings on each question, usually highlighting the most frequent answer given.

The results for questions $03,05,06,11,14$, and 16 are shown in Figure 2.

In relation to $03,61 \%$ of the respondents declared that sometimes they realize that they conducted an AVSEC procedure with error and try to correct it.

In relation to $05,58 \%$ of the AVSEC professionals answered that they never/sometimes advise a coworker who is operating the $\mathrm{X}$-ray equipment about the situation of the lines, when they are working with flow control in hours of great flow of passengers.

In relation to $06,63 \%$ of the respondents declared that they always recognize that the sound alarm activated by the detector comes from the security checkpoint, in hours of great flow of passengers.

In relation to $11,53 \%$ of the respondents affirmed that they always accomplish the AVSEC procedures when they refer to authorities.

In relation to $14,35 \%$ of the professionals revealed that they always indicate they need to be attentive when they are operating the X-ray equipment and a coworker talks to them.

In relation to $16,46 \%$ of the participants answered that always/most of the time the experience they have in AVSEC prevails over the regulation procedures provided by the Brazilian civil aviation authority, ANAC.

The results for question number 20 are shown in Figure 3.

In relation to 20 , for $62 \%$ of the participants, the level of attention is high/total in the identification of a prohibited object or an object with controlled access, through the Xray equipment, even when a coworker insists on talking to them. 


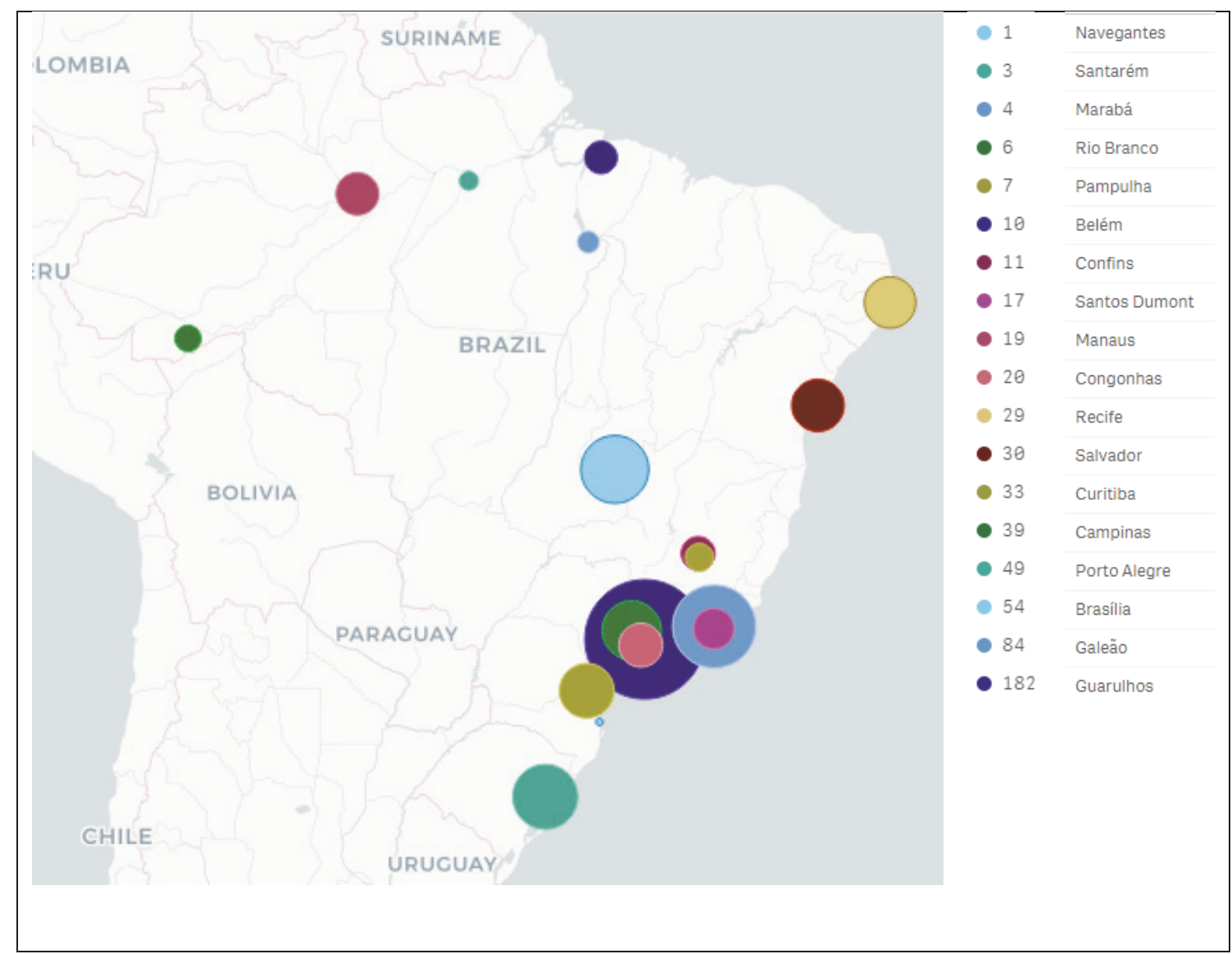

FIGURE 1: Distribution of questionnaire respondents, over 18 Brazilian airports in the years of 2013 and 2014.

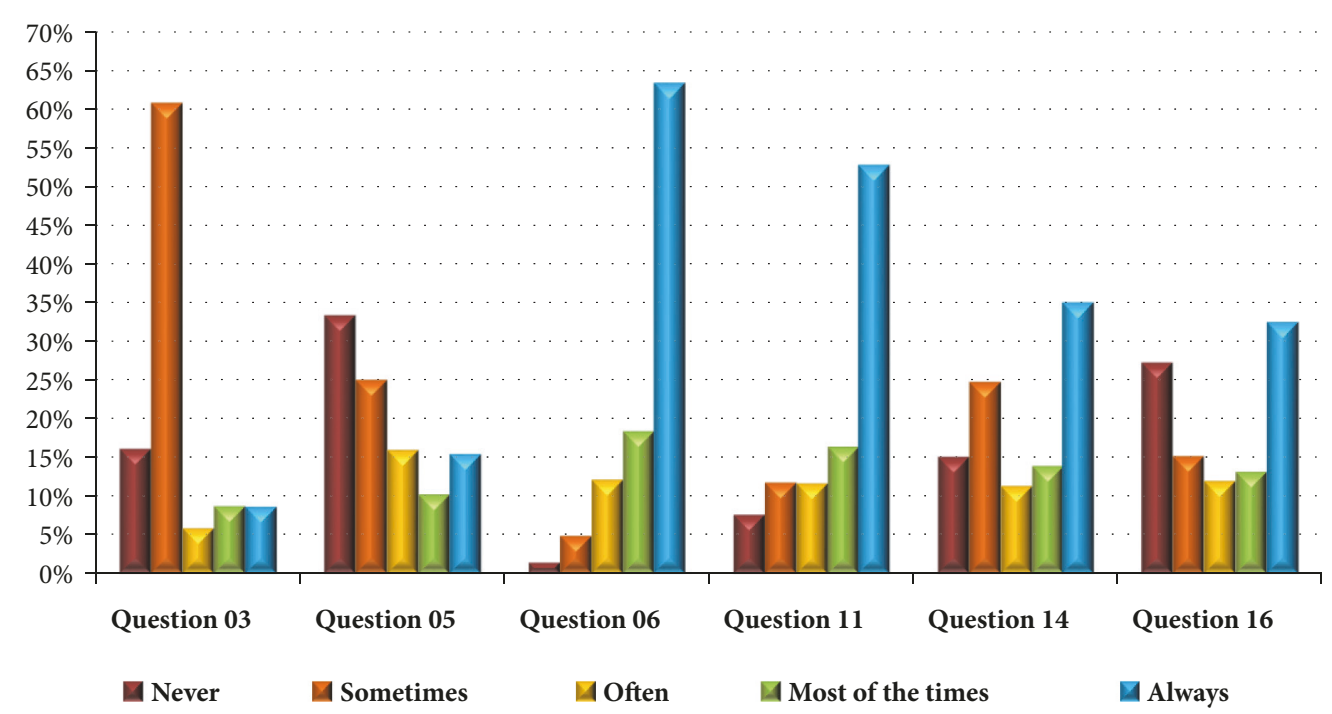

FIGURE 2: Relative frequency of answers to six questions of the questionnaire, in the frequency scale.

The frequencies of variables in the agreement scale, concerning questions $36,39,40,50,57,58$, and 59 , are shown in Figure 4.

In relation to $36,39 \%$ of the participants declared that they agree/totally agree that they are seeking another job to supplement the income, although they like working with AVSEC.

In relation to $39,64 \%$ affirmed that they agree/totally agree that there is turnover of personnel in the airport they work. 


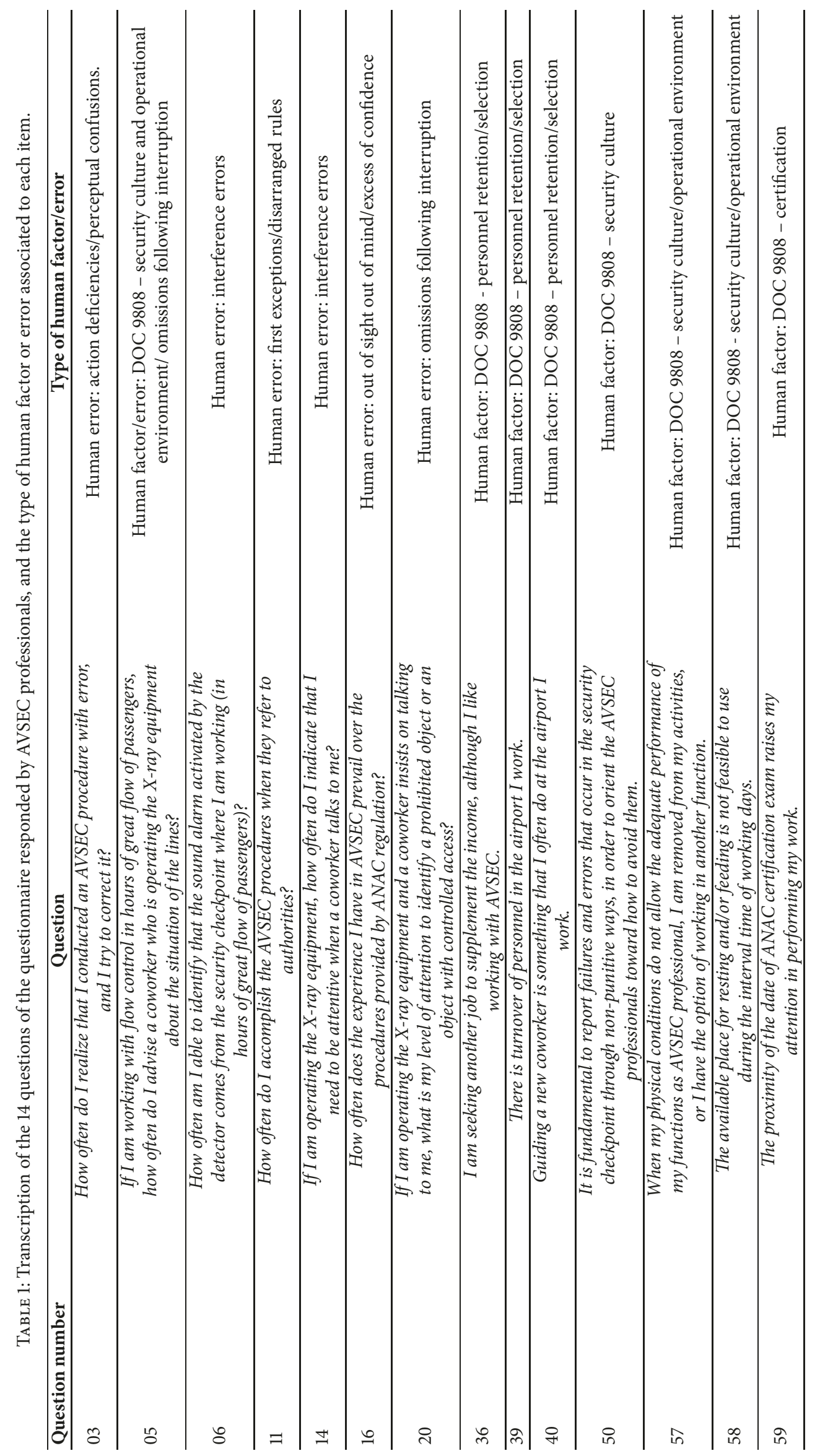




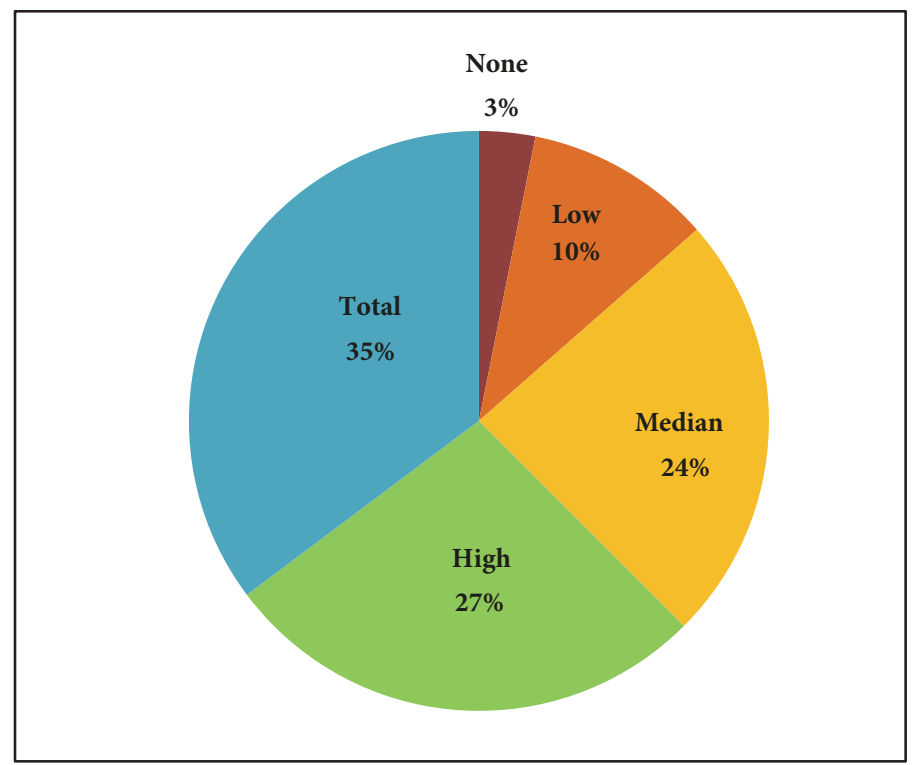

Figure 3: Relative frequency of answers to question 20 of the questionnaire, in terms of level of attention (low, median, or high).

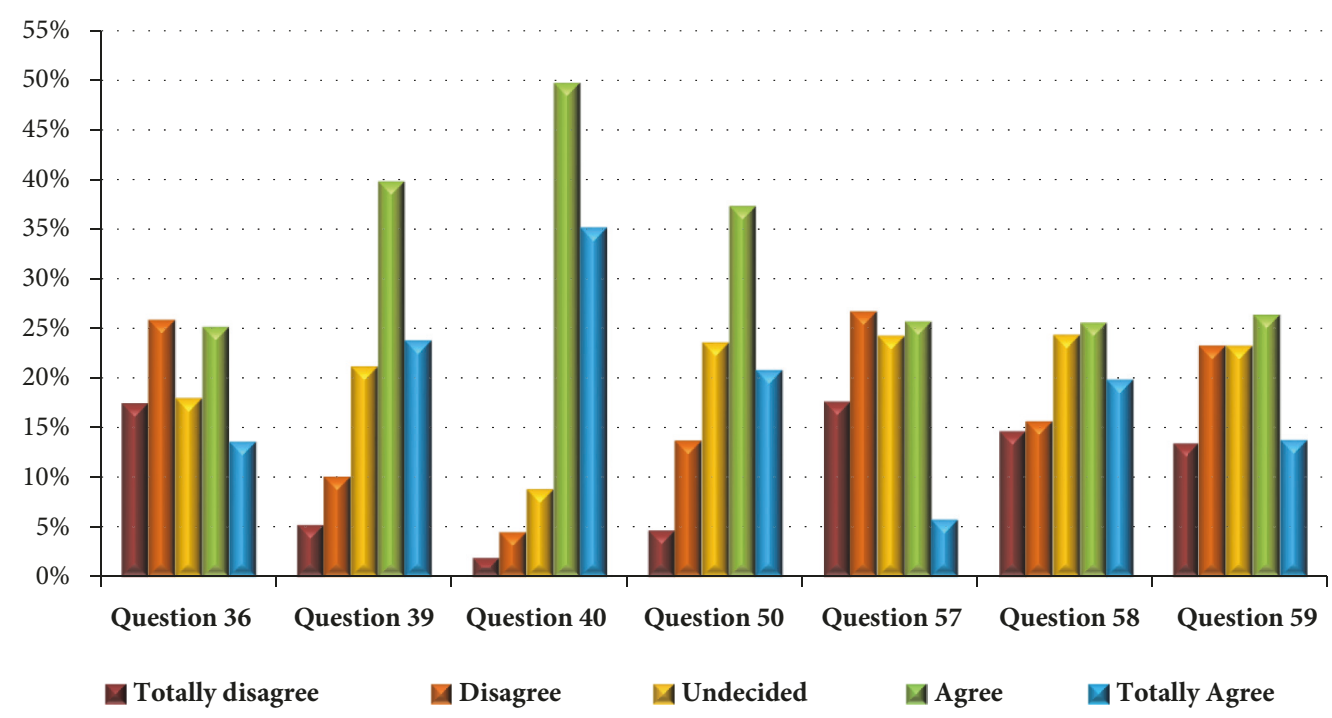

FIGURE 4: Relative frequency of answers to seven questions of the questionnaire, in the agreement scale.

In relation to $40,50 \%$ of the respondents declared that they agree that guiding a new coworker is something they often do at the airport they work.

In relation to 50,58\% of the professionals affirmed that they agree/totally agree that it is fundamental to report failures and errors that occur in the security checkpoint through nonpunitive ways, in order to orient the AVSEC professionals toward how to avoid them.

In relation to $57,45 \%$ of the participants revealed that they disagree/totally disagree that when their physical conditions do not allow them to perform adequately their AVSEC professional duties, they are either removed from the activities, or they have the option of working in another function.

In relation to $58,46 \%$ of the respondents declared that they agree/totally agree that it is not feasible to use the available place for resting and/or feeding during their breaks in working days.

In relation to $59,40 \%$ of the professionals answered that they agree/totally agree that the proximity of the ANAC certification exam date raises their attention in performing their work.

\section{Discussion and Conclusion}

The intervention in the context of aviation security, under an ergonomic perspective and with the objective of decreasing the incidence of human factors and errors in the security checkpoint of airports, is based on the assumption that the professionals involved in real work situations are not identical. They bring their experiences, strategies, and representations and use them to perform security screening process. 
Thus, as mentioned by [9], security has to be considered as a common goal and absolute good that exists alongside quality and safety.

This study has approached some concepts of mental processes related to real work situations, which involve actions and decisions, to better understand the failures in the security checkpoint that result from these processes. Therefore, several areas of Psychology were covered, such as human cognition, perception processes, and cognitive processes.

After analyzing data from 40 events in X Airline Company over two years, [10] found that the highest percentage of key factors contributing to errors were the repetitive and monotonous jobs, the work procedures that were not followed, and the complacency. The work on the security checkpoint may be repetitive, but unlikely to be monotonous, considering the dynamics of the activity.

Attention is one of the factors that influence the labor actions. According to [2], attention correlates with the amount of stimuli existent in the situation of work and may be classified into four types: selective, which defines the focus of attention, emphasizing or disregarding the stimuli; surveillance, which waits passively for stimuli from the environment; survey, which refers to the active search for evidence, signals, and stimuli of the context; and divided attention, which allocates resources to more than one labor activity.

Moreover, in the technical document DOC 9683 [11], it was stated that attention has two meanings: selective attention, which is related to the capacity of human being to concentrate on a source of information, which can merge with other sources; divided attention, which considers the capacity to divide the attention in more than one stimulus, synchronously.

The knowledge about the concept and types of attention contributes to the comprehension of how the AVSEC professional processes certain information, establishes priorities, and uses them to perform the functions in the security checkpoint. Our results on variable 14 showed that $35 \%$ of the respondents always indicate they need to be attentive when a coworker talks to them while they are operating the X-ray equipment (Figure 2). It raises the possibility of an inefficient supervision or the lack of more effective labor instructions.

Direct attention is necessary, not only to realize and process the vulnerabilities, but also to make decisions and give the right responses. In complex and dynamic systems, the demands for attention result from the overload of information, complex decision-making, and multiple tasks, which can rapidly overcome the limited attention capacity of a person [10].

Although the activities in the security checkpoint are previously defined, such as the control of passenger flow, the manual screening process of passengers and luggage, and the security screening process, there are situations in which the AVSEC professional needs to execute two or more of these activities, simultaneously. For instance, while a flow controller keeps their attention in performing their main tasks, they can also come across with a passenger who makes many questions and demands part of his attention.
In other words, the capacity of simultaneous processing of different information and the establishment of priorities are required from the professional. Depending on the limits of this capacity, human factors and errors may be taken into account in screening procedures.

Variable 20 data (Figure 3 ) revealed that, even if a member insists on talking to $\mathrm{h}$ the $\mathrm{X}$-ray equipment operator, the attention level of this operator is high/total, according to the answers of $62 \%$ of the participants. Thus, the involuntary negligence on identifying prohibited items presented a low representativeness. Still, this index could be increased by formulating more precise labor instructions and establishing more efficient ways of supervision.

We have also investigated the human error called by Reason [12] "out of sight out of mind", by going through variable 16. The answers of $46 \%$ of the professionals demonstrated that experience in AVSEC always/most of the time prevails over the Brazilian civil aviation authority, ANAC, regulation (Figure 2). Despite this result, it is crucial to emphasize that the ANAC regulations legally support AVSEC professionals work. It must seek the aligning between the efficacy level and the applicability of rules and procedures. And since complying with such regulation is compulsory, supervisors and other control agents should always be requested to comply with them.

As far as the AVSEC professionals acquire more experience and knowledge in the process of security screening, it is possible that they automate some steps of their activities. As described by [2], the automating implies leaving a controlled situation, which employs much attention to the action, and entering in a situation without need of control, which demands only moderate attention and efforts and allows saving of cognitive processing. In the automation context, the AVSEC professionals may execute part of their tasks with less cognitive effort, demand lower amounts of knowledge, and perform different activities simultaneously. However, if the activities are concurrent, being similar in their elements and requiring the same perceptive senses or the same type of reasoning, they may affect the overall performance of the professional.

The discussion on the incidence of error in relation to experience is also portrayed by [10]. The experts with several years of work made more errors (e.g., errors of commission and omission) because of complacency and for not following work procedures.

Furthermore, automation is characterized as attentional and does not occur under the total control of the individual. Several times, the AVSEC professionals may direct their attention to stimuli when they do not intend to, or do not depend on their own wish. It may happen, for instance, during the screening process of celebrities, public personalities, or clarifying doubts of the passengers.

The answers to the variable 11 showed that $20 \%$ of the respondents affirmed that they never/sometimes accomplish the AVSEC procedures when they refer to authorities (Figure 2). This may indicate that complacent labor environments, tending to be noncompliant with AVSEC procedures, or environments that tolerate exceptionalities out of the standard procedures may be leading to the infringement of 
rules. Taking into account its expressiveness, this is bound to raise Brazilian authorities awareness on AVSEC.

This study does not intend to reject the roles played by automation, but rather to improve the understanding of the elements that can unleash them, causing the incidence of human factors and errors that compromise the process of security screening. Therefore, it is relevant to investigate if the nature of existing stimuli at the security checkpoint can facilitate, or interfere with, the conscious control of a required action. Some examples of these facilitating stimuli are the sound alarms that draw the attention of the professional or the various possibilities of image contrasts provided by X-ray equipment, among others.

We have also approached the human error classified as action deficiencies, or perceptual confusion, by going through variable 03 . The results showed that $61 \%$ of the respondents declared that sometimes they realize the conduction of an AVSEC procedure with error and try to correct it (Figure 2). This data suggests that, almost always, the correction of procedures in the security checkpoint is inserted in a context of unfeasibility. Once the screening process has been done, the passengers and their belongings have already accessed the security restricted area (SRA) or entered in the aircraft. Thus, actions to mitigate an error in this critical point of the airport must be provided in risk management programs, by air operators or the airport's operator.

In labor conditions, the professionals understand and organize the information (cognitive processes) in a correlated way with the perceptual processes. According to [2], "[...] the perception is a set of processes by means of them we receive, recognize, organize and understand the sensations received from the environmental stimuli". In the DOC 9683 [11], it was mentioned that the perception, or the information processing, may be disrupted in distinct ways. The labor environment in which the information capture is altered, confused or disturbed may induce the occurrence of errors and vulnerabilities to the system, since it complicates the information comprehension by the professionals and burdens them with the frequent need to make corrections.

Practical and science-based approaches to improve threat detection at airports have been proposed [13]. Among them, there is the optimization of the roles played by human operators. Since they have special capabilities needed for alarm resolution, such as pattern recognition, abstract reasoning, spatial visualization, and cognitive flexibility, their role in the resolution of detected signals should be strengthened. However, even in this role, the combined effects of the design of the specific operator tasks, the environment in which the tasks are performed, and the selection and training of operators would be critical to the realization of effective performance. Therefore, using human operators appropriately in threat detection systems would require systematic efforts to match their capabilities with the requirements of the system. It could also be used to minimize the effects of human limitations.

In the environment, when the stimuli are detected through perception, the cognitive processes are activated, as well as the search of either problem resolution or decisionmaking, in a continuous and integrated manner. Moreover, the professionals evoke memories of other situations and similar concepts, which may help them to comprehend a problem or to justify some actions. The problem resolution is related to different elements of a situation and to the ways that individuals use the knowledge to construct a mental representation of the problem and to generate solutions [2]. Therefore, the clarity of information, the previous experience of AVSEC professionals, and their capacity to use competence and attention may increase their possibilities of a proper decision-making in the face of a problem.

On the other hand, a professional may select wrongly an inappropriate model from the labor memories. Based on a subset of situational clues, he may use this model to interpret the perceived data and to make decisions [14]. For instance, when the AVSEC professional is demanded to accelerate his decision-making to release a passenger to the security restricted area, this procedure may occur in a partial and fragmented way, causing failures and human factors or errors. From the perspective of [7], these aspects would be classified as action deficiencies, due to the use of unadvisable rules.

The type of human error caused by interferences was investigated in variable 06 . We identified a low frequency of $6 \%$ of the participants, who affirmed that they never/sometimes recognize that the sound alarm activated by the detector comes from the security checkpoint, in hours of great flow of passengers (Figure 2). It demonstrates the sound emitted by the detector has high level of efficiency and reaches its objective of supporting the work of AVSEC professionals.

It is known that complex cognitive tasks, which concern national security matters, such as the security screening process, are often subject to several restrictions of time. These restrictions can be formally established or occur due to pressure for achieving goals, for instance, as a result of limited time of the aircraft on the ground or in specific circumstances, such as the peak hours in the airports. It becomes primordial to control these restrictions, avoiding the possibilities of human factors and errors of great coverage.

The human factor or error called security culture and operational environment, or omission following interruption, has been discussed in the technical document DOC 9808 [8] and approached by variable 05 . The answers of $58 \%$ of the respondents showed that when they are controlling the flow of passengers, they never/sometimes advise the operator of X-ray equipment about the situation of the lines (Figure 2). This result may suggest that involuntary suppression of some steps in the process of security screening, which is caused by interruptions, can result from a necessity of fast processing of passengers, a security culture with less solidity in AVSEC, or still a labor environment which does not balance the demands of air transport users and the available human and material resources.

Concerning the organization's security culture, [15] emphasized its importance in a study on information technology (IT) security. They described an IT risk management model, which was applied to an academic institution's information system and focused on the breaches and risks related to the human factor. The results of the model's application supported the claim that information security officials tend to overinvest in security technological tools and to underinvest 
in security workplace culture improvements. As reported by them, security officers place more emphasis on technological tools rather than improving the workplace culture. Our study group is currently developing a specific research on security culture to discuss their effects in the security checkpoint operations.

In addition, another aspect that affects the performance at work is motivation. Conceptually, motivation for working reflects the difference between what a professional can do and what they will really do. It is what stimulates or encourages a person to behave in a determined manner [11]. The relationship between expectation and reward can also be considered as a motivating factor. The benefit of a reward and the subjective probability of reaching it determine the level of effort that will be applied to earn a certain reward. Thus, the more motivated the AVSEC professionals, the higher their performance levels at work.

Another motivational factor that aims at the identification, recognition, and correction of human factors and errors could be established through a notification system. This system is necessary to promote a security culture and working environment in which all AVSEC professionals feel safe to report their observations about behaviours that represent deviations from the regulations. Thus, it is essential to establish a nonpunitive and blame-free culture to encourage contributions, both from the individual and the organization. This importance is also recognized by the authors of [16], who reported that the problem of coordination or communication is the main factor that leads to errors during information cognitive process, in order to identify how human ability contributes to the design of technology and to understand the connections within human information processing and human performance.

The knowledge derived from reporting systems provides the basis to collect, document, and process information related to the existence of human errors, to enhance the effectiveness of the screening process [6]. Using the information available on the basis of reports arising from screeners' work every day may avoid a latent failure in the system and may promote the improvement of ergonomics of the activity.

The findings of [17] suggested that, to reduce errors related to information cognitive skills, the most efficient way is to solve the coordination, communication, and planning problems. Once more, the importance of implementing a notification system was demonstrated.

We investigated the human factor classified by the DOC 9808 [13] as personnel retention/selection, through the variables 36, 39, and 40 (Figure 4). Concerning the variable 36, the results revealed that $39 \%$ of the participants declared that they agree/totally agree that they are seeking another job to supplement the income. As previously identified in [5], the income earned by the AVSEC professionals is a factor that discourages their retention in the career and is not compatible with their responsibilities and the complexity of their attributions.

In relation to variable $39,64 \%$ of the respondents affirmed that they agree/totally agree they there is turnover of personnel in the airport they work. This turnover is mainly associated with the low salaries, inexistence or discontinuity of incentive policies of personnel retention, lack of labor valorization, labor characteristics, and other aspects with which the AVSEC professional may not adjust over the years. We emphasize the importance of fostering policies for the retention of AVSEC professionals, since high levels of turnover of these professionals imply high number of individuals with access to AVSEC subjects and knowledge on their applicability in civil aviation, especially their fragilities. Consequently, controlling the turnover indexes may reduce risks for the civil aviation security system against acts of unlawful interference.

Moreover, the answers to the variable 40 showed that $50 \%$ of the professionals agreed that guiding a new coworker is something that they often do at the airport. This outcome is related to the answers to the variable 39 , concerning the turnover of AVSEC personnel. In addition, the increased demand of passengers and the recent airport concessions in Brazil have prompted an increment of technological and human resources to the civil aviation system.

The human factor classified by the DOC 9808 [8] as security culture/operational environment was investigated through the variables 57 and 58 (Figure 4). With respect to variable 57 , we found that $45 \%$ of the participants revealed that they disagree/totally disagree that when their physical conditions do not allow them to perform adequately the functions of AVSEC professional, they are removed from the activities, or they have the option of working in another function. Concerning variable $58,46 \%$ of the respondents declared that they agree/totally agree that it is not feasible to use the available place for resting and/or feeding during their breaks in working days.

Therefore, an organizational culture considers the physical welfare of its professionals, as life quality programs and other subjects related to ergonomics of the activities. These actions may cause the acquisition of higher levels of labor performance. For this, the infrastructure, the functioning of workstations, equipment, and functions to be performed must be considered.

According to [2], the posture of the AVSEC professional at the security checkpoint exposes various aspects, such as the workstation layout, stereotyped way to settle the body in the physical space, seat, monitor screen, available equipment, comfort level, functions, reactions to the labor requirements, physiological and perceptual aspects, and characteristics of the individuals. Moreover, concerning the X-ray equipment, some characteristics of monitor screens are desirable: they should respect the operator's height, avoid the upper part of screen being above or below the eye line, ensure a distance of 50 to $70 \mathrm{~cm}$ between the eyes and the screen, and increase this distance when the image size is increased, to minimize visual efforts.

In this context, the body stiffness, prolonged muscle contraction, and working at a constant standing position may unleash human factors or errors, causing the decrease in pace of work, or a temporary deficit of attention, and the overload in information processing, since the professional receives constant alerts about his uncomfortable posture.

We have also investigated the human factor classified by the DOC 9808 [8] as security culture, through the 
variable 50 (Figure 4). The data showed that $58 \%$ of the professionals affirmed that they agree/totally agree that it is fundamental to report failures and errors that occur in the security checkpoint of nonpunitive ways, in order to guide the AVSEC professionals toward how to avoid them. It confirms the theoretical argument presented in the DOC 9808 [8], about the importance of implementing a notification system. Regarding the relevance of reporting failures, many researchers currently stress that insider human factor is the greatest cause of security breaches [15].

Furthermore, the answers to variable 59 (Figure 4) refer to the human factor classified by the DOC 9808 [8] as certification. We verified that $40 \%$ of the professionals answered that they agree/totally agree that the proximity of ANAC certification exam dates raises their attention in performing their duties. On the other hand, $13 \%$ of the professionals totally disagreed with this statement. These results highlight the assertive contribution of the certification process of AVSEC professionals to the attention levels and labor performance.

As reported by [18], some assessments called Work or Job Safety Analyses are aimed at protecting the workforce from injury, rather to protect the public at large and the environment from a major catastrophe. However, according to the human error theory [12], the error types are fundamentally the same, whether in the home or in a factory, or in a nuclear power plant. At a philosophical level, therefore, such error analysis tools should also have relevance to less complex systems.

In conclusion, we emphasize the importance of understanding how the professionals realize and behave towards the information assimilated at the labor environment, especially in complex systems and in contexts of defense in depth, such as the security screening process.

\section{Data Availability}

The data used to support the findings of this study are available from the corresponding author upon request.

\section{Conflicts of Interest}

The authors declare that there are no conflicts of interest regarding the publication of this paper.

\section{Acknowledgments}

The authors of this study express thanks to all who participated in this research for their cooperation; to the National Civil Aviation Agency (ANAC), which supported the data release; and to their colleague Luciano Lopes de Azevedo Freire for all of his contributions.

\section{References}

[1] A. Schwaninger, D. Hardmeier, and F. Hofer, "Measuring visual abilities and visual knowledge of aviation security screeners," in Proceedings of the Proceedings - IEEE 38th Annual 2004
International Carnahan Conference on Security Technology, pp. 258-264, USA, October 2004.

[2] J. E. A. Abrahão, Introdução à ergonomia: da prática à teoria, Blucher, São Paulo, Brazil, 2009.

[3] M.-C. Hsieh, M.-C. Chiu, and S.-L. Hwang, "An optimal range of information quantity on computer-based procedure interface design in the advanced main control room," Journal of Nuclear Science and Technology, vol. 52, no. 5, pp. 687-694, 2015.

[4] Y. Wiseman and Y. Giat, Multimodal Security in Passenger and Freight Transportation: Frameworks and Policy Applications, D. Rhoades, G. Reniers, and J. Szyliowicz, Eds., Edward Elgar Publishing, 2016.

[5] M. S. Arcúrio, J. A. Fortes, and T. Armborst, "Fatores e erros humanos na inspeção de segurança da aviação civil brasileira," Revista Psicologia, Organizações e Trabalho, vol. 16, no. 3, pp. 259-273, 2016.

[6] M. S. F. Arc, E. S. Nakamura, R. R. D. Pereira, T. Armborst, and J. Fortes, "Security screening process of passengers in Brazilian airports: Bivariate analysis of human errors and factors," Journal of Airport Managemente, vol. 11, no. 3, pp. 271-293, 2017.

[7] J. Reason, El Error Humano, Modus Laborandi, Madrid, Spain, 2009.

[8] Organización de Aviación Civil Internacional, DOC 9808: Los factores humanos em las operaciones de seguridad de la aviación civil, Montreal, Québec, Canada, 2002.

[9] M. B. Salter, "SeMS and sensibility: Security management systems and the management of risk in the Canadian Air Transport Security Authority," Journal of Air Transport Management, vol. 13, no. 6, pp. 389-398, 2007.

[10] G.-F. Liang, J.-T. Lin, S.-L. Hwang, E. M.-Y. Wang, and P. Patterson, "Preventing human errors in aviation maintenance using an on-line maintenance assistance platform," International Journal of Industrial Ergonomics, vol. 40, no. 3, pp. 356$367,2010$.

[11] Organización de Aviación Civil Internacional, DOC 9683: Manual de Instrucción sobre Factores Humanos, 1998.

[12] J. Reason, Human Error, Cambridge, United Kingdom, Cambridge University Press, United Kingdom, 1990.

[13] D. H. Harris, "How to really improve airport security. Ergonomics in design: the quarterly of human factors applications," Ergonomics in design: the quarterly of human factors applications, vol. 10, no. 1, pp. 17-22, 2002.

[14] R. Dismukes, Human Error in Aviation.Critical Essays on Human Factors in Aviation, Ashgate, 2009.

[15] M. Dreyfuss and Y. Giat, "A risk management model for an academic institution's information system," Information Resources Management Journal, vol. 31, no. 1, pp. 83-96, 2018.

[16] C. H. J. Wickens, Engineering Psychology and Human Performance, Prentice Education Taiwan Ltd Press, 2002.

[17] M.-C. Chiu and M.-C. Hsieh, "Latent human error analysis and efficient improvement strategies by fuzzy TOPSIS in aviation maintenance tasks," Applied Ergonomics, vol. 54, pp. 136-147, 2016.

[18] B. Kirwan, "Human error identification techniques for risk assessment of high risk systems - Part 2: Towards a framework approach," Applied Ergonomics, vol. 29, no. 5, pp. 299-318, 1998. 


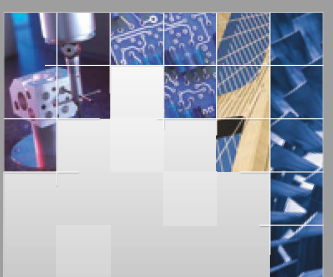

\section{Enfincering}
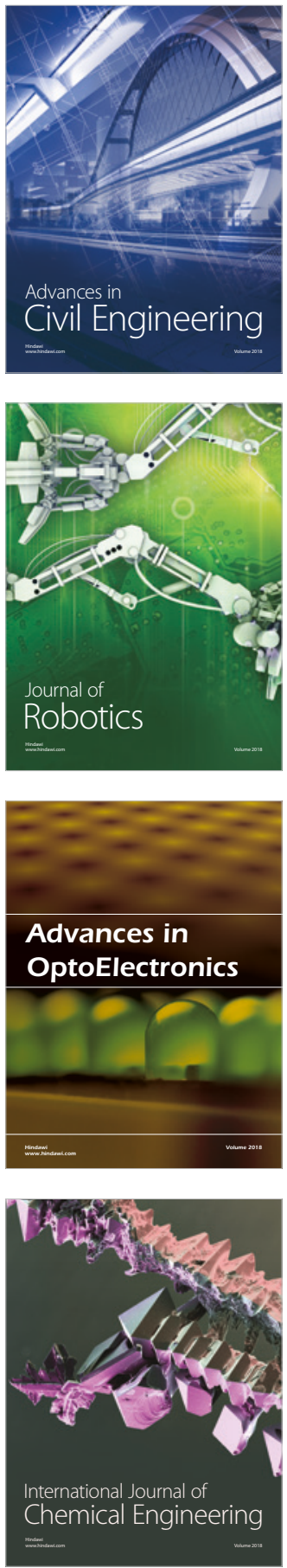

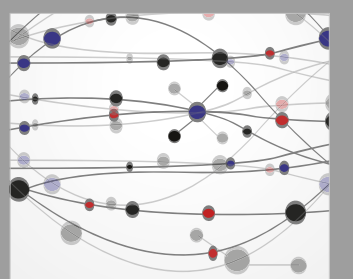

\section{Rotating \\ Machinery}

The Scientific World Journal

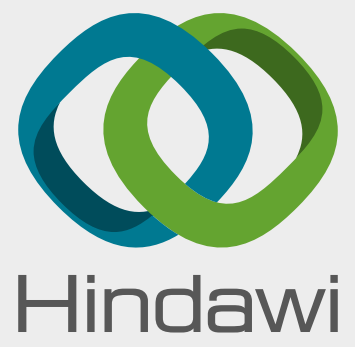

Submit your manuscripts at

www.hindawi.com
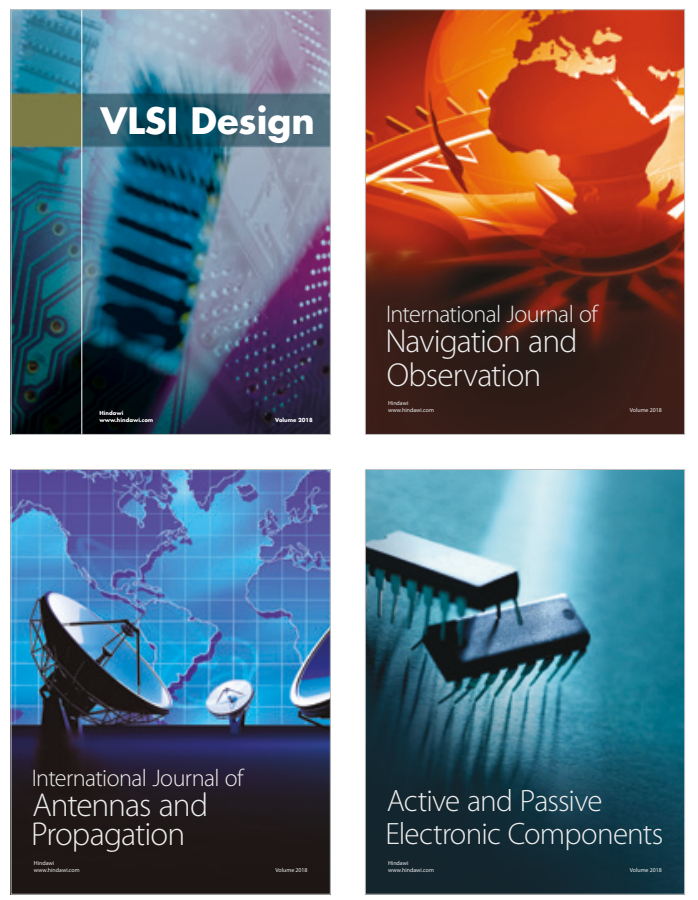
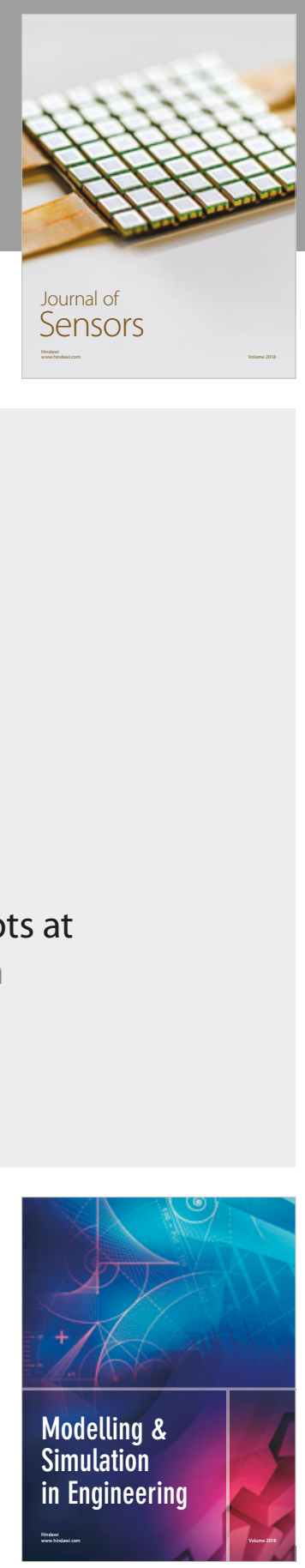

\section{Advances \\ Multimedia}
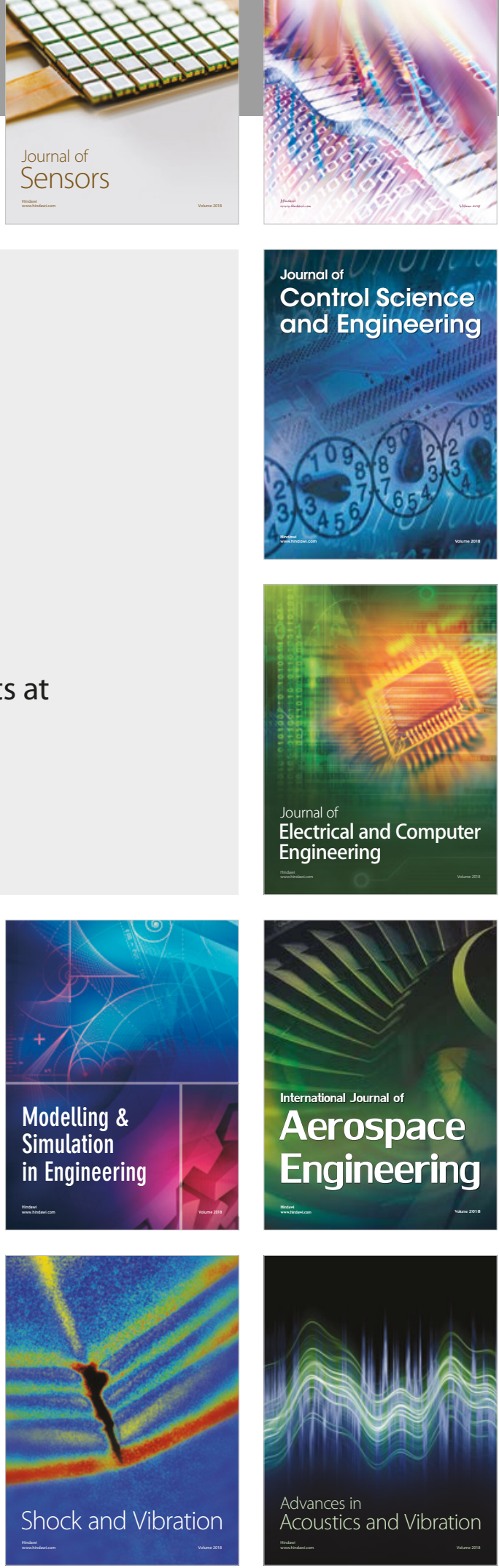